\title{
Dynamic model of a DC-DC quasi-Z-source converter (q-ZSC)
}

\author{
Muhammad Ado $^{1}$, Awang Jusoh ${ }^{2}$, Abdulhamid Usman Mutawakkil ${ }^{3}$, Tole Sutikno ${ }^{4}$ \\ ${ }^{1,2}$ School of Electrical Engineering, Universiti Teknologi Malaysia, Malaysia \\ ${ }^{1,3}$ Bayero University Kano, Nigeria \\ ${ }^{4}$ Department of Electrical Engineering, Universitas Ahmad Dahlan, Indonesia
}

\begin{tabular}{l} 
Article Info \\
\hline Article history: \\
Received Aug 10, 2018 \\
Revised Nov 20, 2018 \\
Accepted Dec 11, 2018 \\
\hline
\end{tabular}

Keywords:

Buck-boost converter

Dynamic model

Impedance source

Q-ZSC

Small signal model

\begin{abstract}
Two quasi-Z-source DC-DC converters (q-ZSCs) with buck-boost converter gain were recently proposed. The converters have advantages of continuous gain curve, higher gain magnitude and buck-boost operation at efficient duty ratio range when compared with existing q-ZSCs. Accurate dynamic models of these converters are needed for global and detailed overview by understanding their operation limits and effects of components sizes. A dynamic model of one of these converters is proposed here by first deriving the gain equation, state equations and state space model. A generalized small signal model was also derived before localizing it to this topology. The transfer functions (TF) were all derived, the poles and zeros analyzed with the boundaries for stable operations presented and discussed. Some of the findings include existence of right-hand plane (RHP) zero in the duty ratio to output capacitor voltage TF. This is common to the Z-source and quasi-Z-source topologies and implies control limitations. Parasitic resistances of the capacitors and inductors affect the nature and positions of the poles and zeros. It was also found and verified that rather than symmetric components, use of carefully selected smaller asymmetric components L1 and C1 produces less parasitic voltage drop, higher output voltage and current under the same conditions, thus better efficiency and performance at reduced cost, size and weight.
\end{abstract}

Copyright $(2) 2019$ Institute of Advanced Engineering and Science. All rights reserved.

\section{Corresponding Author:}

Awang Jusoh,

School of Electrical Engineering,

Universiti Teknologi Malaysia,

81310 Johor Bahru, Malaysia.

Email: awang@utm.my

\section{INTRODUCTION}

Impedance source converters (ZSC/ISC) couple converter's main circuit to its power source [1]. They provide additional features not obtained in prior current fed or voltage fed converters such as dead or overlap time in addition to their advantages [2].

Applicability of Z-source concept to ac-ac [3]-[5], ac-dc [6], dc-ac [7]-[12] and dc-dc [13], [14], [23], [15]-[22] power conversion generated a lot of interest and research resulting in the development of variant and new topologies [24]. First application of ZSC was the ZSI for fuel cell application [1] then drives [4].

Reference [25] proposed a modified impedance source converter (ZSC) called quasi-ZSC (q-ZSC) shown in Figure 1 by swapping the positions of switches and inductors to solve problems like discontinuous input current, high capacitor voltage requirement for the voltage fed ZSCs and high inductor current requirement for current fed ZSCs. Most of early ZSC and q-ZSCs [4], [26], [35]-[40], [27]-[34] focused on inverter applications except [5] on ac-ac converter and [6] on rectifiers. Reference [13] extended ZSC and q-ZSC concept to DC-DC applications by proposing four non-isolated DC-DC ZSC and q-ZSC topologies each, then [20], [22] proposed isolated DC-DC ZSCs after which several other isolated and non-isolated DC-DC converter topologies have been proposed. 
The major difference between impedance source dc-ac (inverters) and DC-DC converters lies on how the output is taken. For inverters, it is taken across a switch while for the DC-DC converter, they are mostly taken across a capacitor [13] as shown in Figure 1 (b) and (c), although [15], [16], [18], [19], [21]-[23] took the output across a switch albeit with additional components in what is called PWM DC-DC impedance source converters. Reference [15] analysed the steady-state performance of such converters in continuous conduction mode (CCM).

References [29], [41]-[45] applied state space averaging [46] and Taylor's series expansion and derived the small signal analysis to investigate the dynamic characteristics of different ISI topologies. Accurate small signal model is needed to obtain a global and detailed overview of system dynamics by understanding system limits and components sizes [44]. It is based on the assumption of perturbations around steady-state operating point [47]. Small signal perturbations (e.g. $\left.\tilde{\mathbf{d}}(\mathbf{t}), \tilde{\mathbf{v}}_{\mathbf{g}}(\mathbf{t}), \tilde{\mathbf{i}}_{\mathbf{g}}(\mathbf{t})\right)$ are applied to the steady state duty ratio (D) and input variables (e.g. $\mathrm{Vg}$ and $\mathrm{Ig}$ ) to obtain the small signal model. These perturbations causes the dynamic state variables (e.g. $\mathbf{v}_{\mathbf{C} 1}, \mathbf{v}_{\mathbf{C} 2}, \mathbf{i}_{\mathrm{L} 1}$ and $\mathbf{i}_{\mathrm{L} 2}$ ) to vary (by $\tilde{\mathbf{v}}_{\mathbf{C} 1}, \tilde{\mathbf{v}}_{\mathbf{C} 2}, \tilde{\mathbf{i}}_{\mathbf{L} 1}$ and $\tilde{\mathbf{i}}_{\mathbf{L} 2}$ respectively).

Use of small signal models to obtain dynamic models for controller design makes them very important. They are also used to obtain the transfer functions between state variable and system input by assuming other system inputs to be zero [41], [44], [48].

Interestingly, the existing publications on dynamic models of ISCs [41], [44], [45], [48]-[51] focused on inverters. This is mainly due to the fact highlighted by [15] that majority of the literature on ISCs focuses on the inverter mode of operation although [52] worked on PWM DC-DC converter. DC-DC ZSC/q-ZSCs are not very popular due to common deficiencies like lack of buck-boost capability at the efficient duty ratio range of [0.35 to 0.65] [53], discontinuous gain curve and higher components count as compared with the traditional buck-boost converter (BBC).

However, more findings are making ISCs overcome these challenges such as [54], [55] where the gain and continuous gain curve of BBC were achieved using non-isolated q-ZSC topologies. These topologies produced higher magnitude output voltages and currents than the corresponding buck-boost converters thus giving them potential advantages.

In this paper, the concept of dynamic modelling is extended to the DC-DC q-ZSC. This Extension is important because their applicability is increasing while there are no or very few existing dynamic models of them.

The modelling began by first considering an ideal circuit to derive the ideal gain equation. Next, nonsymmetric, real components were considered rather than the simple symmetric or ideal q-ZSC. The use of nonsymmetric components allows identifying the individual effect of each component while non-ideal components allow analyzing the effects of the parasitic resistances of the components.

As common to circuits that change over switching cycle, state space averaging [46] was used to describe the circuit. State space averaging requires generating sets of equations, with each representing a switching state [47] and then averaged over the switching period.

ISCs can be controlled with or without shoot-through [34] or open state. This converter was controlled without using shoot-through or open states in order to enable fair comparisons with the traditional buck-boost converter which is operated using only two switching states (with dead-time) since they have identical gain equation.

Findings from this dynamic modelling show that the parasitic resistances of the capacitors and inductors are among the major factors that determine most of the poles and zeros and circuit efficiency as detailed in the discussion section.

\section{CIRCUIT ANALYSIS}

This section is classified into two: gain derivation and state equations derivation. Circuit analysis was done using ideal and real circuits for the gain and state equations derivation respectively. The analyses were done using two switching modes with respect to $S_{1}$ while $S_{2}$ is complementarily switched with respect to $S_{1}$ giving rise to two operation modes shown in Figure 2. The duty ratio of the modes are ' $\mathrm{D}$ ' and ' $1-\mathrm{D}^{\prime}$ for modes I and II respectively. $\mathrm{C}_{1}, \mathrm{C}_{2}, \mathrm{~L}_{1}$ and $\mathrm{L}_{2}$ are capacitors and inductors with currents $\mathrm{I}_{\mathrm{C} 1}, \mathrm{I}_{\mathrm{C} 2}, \mathrm{I}_{\mathrm{L} 1}$ and $\mathrm{I}_{\mathrm{L} 2}$, and parasitic resistances $R_{1}, R_{2}, r_{1}$, and $r_{2}$ respectively while $V_{g}, I_{g}, R_{O}$ and $I_{O}$ are input voltage, input current, load resistance and load current respectively.

\subsection{Gain Derivation}

For simplicity, the ideal circuit of Figure 1(b) was used to derive the topology's ideal gain equation by assuming parasitic resistances $R_{1}, R_{2}$ and $r_{1}, r_{2}$ of the capacitors and inductors of Figure 2 to be negligible. 
Mode 1: In this mode as shown in Figure 2(b), $S_{1}$ is ON while $S_{2}$ is OFF. The duty ratio for this mode is D.

$$
\begin{aligned}
& \mathrm{V}_{\mathrm{L} 1}=\mathrm{V}_{\mathrm{O}}-\mathrm{V}_{\mathrm{C} 1} \\
& \mathrm{~V}_{\mathrm{L} 2}=\mathrm{V}_{\mathrm{g}}
\end{aligned}
$$

Mode II: In this mode, $S_{1}$ is OFF while $S_{2}$ is $\mathrm{ON}$ as shown in Figure 2(c). The duty ratio for this mode is $\mathrm{D}^{\prime}=1-\mathrm{D}$.

$$
\begin{aligned}
& \mathrm{V}_{\mathrm{L} 1}=\mathrm{V}_{\mathrm{g}}-\mathrm{V}_{\mathrm{C} 1} \\
& \mathrm{~V}_{\mathrm{L} 2}=\mathrm{V}_{\mathrm{O}}
\end{aligned}
$$

Applying Volt-Second-Balance on $\mathrm{L}_{1}$ and $\mathrm{L}_{2}$ yields

$$
\begin{aligned}
& \bar{V}_{L 1}=D V_{O}+V_{g}-V_{C 1}-D V_{g}=0 \\
& \bar{V}_{L 2}=D V_{g}-V_{O}(D-1)=0
\end{aligned}
$$

From (6),

$$
\mathrm{V}_{\mathrm{O}}=-\frac{\mathrm{D}}{1-\mathrm{D}} \mathrm{V}_{\mathrm{g}}
$$

(7) Is the ideal steady-state output voltage for this converter. It is the same as the ideal steady state output voltage of buck-boost converter where the two switches are switched complimentarily and D is the duty ratio of $S_{1}$ [55].

\subsection{State equations derivation}

The non-ideal circuits of Figure 2 were used to derive the state equations. The circuit's two operation modes are presented in Figure 2(b) and Figure 2(c) and their duty ratios are "D" and "1 - D" for mode I and mode II respectively. $\dot{\mathrm{V}}_{\mathrm{C} 1}, \dot{\mathrm{V}}_{\mathrm{C} 2}, \dot{\mathrm{I}}_{\mathrm{C} 1}$ and $\dot{\mathrm{I}}_{\mathrm{L} 2}$ are the state variables while input voltage $\left(\mathrm{V}_{\mathrm{g}}\right)$, input current $\left(\mathrm{I}_{\mathrm{g}}\right)$, and output current $\left(\mathrm{I}_{\mathrm{O}}\right)$ were chosen as inputs while capacitor voltages $\mathrm{V}_{\mathrm{C} 1}$ and $\mathrm{V}_{\mathrm{C} 2}$, input current $\left(\mathrm{I}_{\mathrm{g}}\right.$ ) and output voltage $\left(\mathrm{V}_{\mathrm{O}}\right)$ as outputs. This is to identify their suitability for controller design as will be revealed by the averaged model.

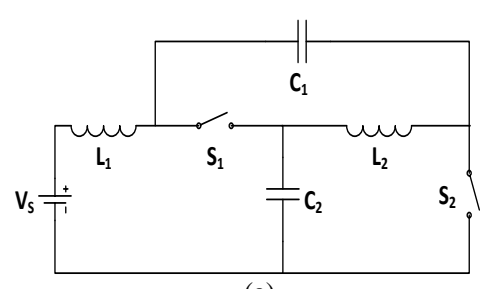

(a)

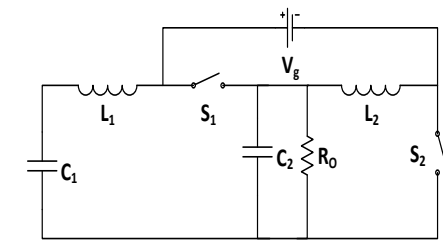

(b)

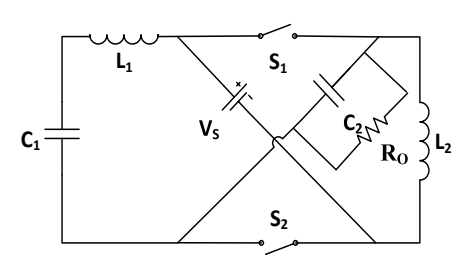

(c)

Figure 1. (a) Generic q-ZSC (b) Derived DC-DC q-ZSC

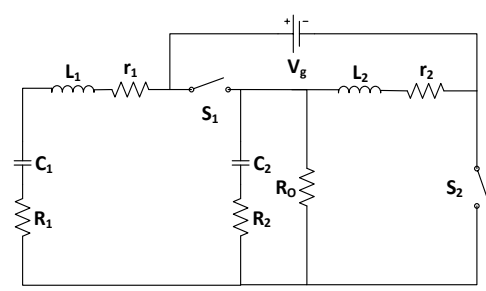

(a)

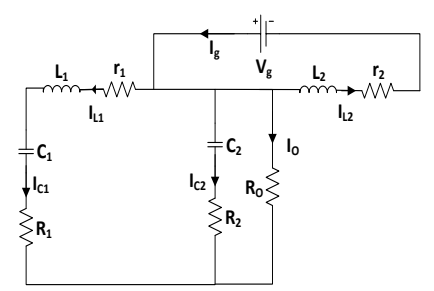

(b)

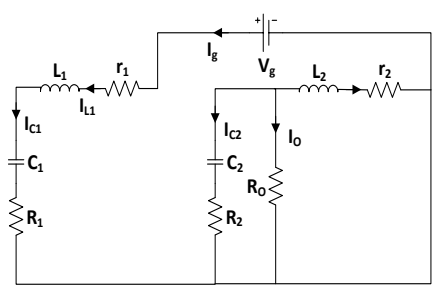

(c)

Figure 2. (a) Considered circuit with parasitic resistances (b) Circuit in mode I (c) Circuit in mode II 
Mode I: In this mode as shown in Figure 2(b), $\mathrm{S}_{1}$ is ON while $\mathrm{S}_{2}$ is OFF. $\mathrm{L}_{2}$ is charged by the input voltage due to the resulting parallel connection. The load, $\mathrm{C}_{1}, \mathrm{~L}_{1}$ and $\mathrm{C}_{2}$ are all isolated from the input voltage. $\mathrm{C}_{1}$ and $\mathrm{L}_{1}$ discharge together to the load while the output filter $\mathrm{C}_{2}$ absorbs the ac ripples. The mode equations are

$$
\begin{aligned}
& \dot{\mathrm{V}}_{\mathrm{C} 1}=\frac{\mathrm{I}_{\mathrm{L} 1}}{\mathrm{C}_{1}} \\
& \dot{\mathrm{V}}_{\mathrm{C} 2}=-\frac{\mathrm{I}_{\mathrm{L} 1}}{\mathrm{C}_{2}}-\frac{\mathrm{I}_{\mathrm{O}}}{\mathrm{C}_{2}} \\
& \dot{\mathrm{I}}_{\mathrm{L} 1}=-\frac{\mathrm{V}_{\mathrm{C} 1}}{\mathrm{~L}_{1}}-\left(\frac{\mathrm{R}_{1}+\mathrm{r}_{1}}{\mathrm{~L}_{1}}\right) \mathrm{I}_{\mathrm{L} 1}+\frac{\mathrm{I}_{\mathrm{O}} \mathrm{R}_{\mathrm{O}}}{\mathrm{L}_{1}} \\
& \dot{\mathrm{I}}_{\mathrm{L} 2}=-\frac{\mathrm{I}_{\mathrm{L} 2} \mathrm{r}_{2}}{\mathrm{~L}_{2}}+\frac{\mathrm{V}_{\mathrm{g}}}{\mathrm{L}_{2}}
\end{aligned}
$$

Expressing in state space form $\dot{\mathrm{X}}_{\mathrm{i}}=\mathrm{A}_{\mathrm{i}} \mathrm{X}+\mathrm{B}_{\mathrm{i}} \mathrm{U}$ where $\mathrm{i}=1$ for mode 1 yields

$$
\dot{\mathrm{X}}_{1}=\left[\begin{array}{c}
\dot{\mathrm{V}}_{\mathrm{C} 1} \\
\dot{\mathrm{V}}_{\mathrm{C} 2} \\
\dot{\mathrm{I}}_{\mathrm{L} 1} \\
\dot{\mathrm{I}}_{\mathrm{L} 2}
\end{array}\right]=\left[\begin{array}{cccc}
0 & 0 & \frac{1}{\mathrm{C}_{1}} & 0 \\
0 & 0 & \frac{-1}{\mathrm{C}_{2}} & 0 \\
\frac{-1}{\mathrm{~L}_{1}} & 0 & \frac{-\left(\mathrm{R}_{1}+\mathrm{r}_{1}\right)}{\mathrm{L}_{1}} & 0 \\
0 & 0 & 0 & \frac{-\mathrm{r}_{2}}{\mathrm{~L}_{2}}
\end{array}\right]\left[\begin{array}{l}
\mathrm{V}_{\mathrm{C} 1} \\
\mathrm{~V}_{\mathrm{C} 2} \\
\mathrm{I}_{\mathrm{L} 1} \\
\mathrm{I}_{\mathrm{L} 2}
\end{array}\right]+\left[\begin{array}{ccc}
0 & 0 & 0 \\
0 & 0 & \frac{-1}{\mathrm{C}_{2}} \\
0 & 0 & \frac{\mathrm{R}_{\mathrm{O}}}{\mathrm{L}_{1}} \\
\frac{1}{\mathrm{~L}_{2}} & 0 & 0
\end{array}\right]\left[\begin{array}{c}
\mathrm{V}_{\mathrm{g}} \\
\mathrm{I}_{\mathrm{g}} \\
\mathrm{I}_{\mathrm{O}}
\end{array}\right]
$$

For the output, $\mathrm{V}_{\mathrm{C} 1}, \mathrm{~V}_{\mathrm{C} 2}, \mathrm{I}_{\mathrm{g}}$ and $\mathrm{V}_{\mathrm{O}}$ are considered and the output equations are

$$
\begin{aligned}
& \mathrm{V}_{\mathrm{C} 1}=\mathrm{V}_{\mathrm{C} 1} \\
& \mathrm{~V}_{\mathrm{C} 2}=\mathrm{V}_{\mathrm{C} 2} \\
& \mathrm{I}_{\mathrm{g}}=-\mathrm{I}_{\mathrm{L} 2} \\
& \mathrm{~V}_{\mathrm{O}}=\mathrm{I}_{\mathrm{O}} \mathrm{R}_{\mathrm{O}}
\end{aligned}
$$

Expressing the output equations in the state space for $Y_{i}=E_{i} X+F_{i} U$ where $\mathrm{i}$ indicates the mode, $\mathrm{i}=1$ for mode 1 and $\mathrm{i}=2$ for mode 2 .

$$
\mathrm{Y}_{1}=\left[\begin{array}{c}
\mathrm{V}_{\mathrm{C} 1} \\
\mathrm{~V}_{\mathrm{C} 2} \\
\mathrm{I}_{\mathrm{g}} \\
\mathrm{V}_{\mathrm{O}}
\end{array}\right]=\left[\begin{array}{cccc}
1 & 0 & 0 & 0 \\
0 & 1 & 0 & 0 \\
0 & 0 & 0 & -1 \\
0 & 0 & 0 & 0
\end{array}\right]\left[\begin{array}{c}
\mathrm{V}_{\mathrm{C} 1} \\
\mathrm{~V}_{\mathrm{C} 2} \\
\mathrm{I}_{\mathrm{L} 1} \\
\mathrm{I}_{\mathrm{L} 2}
\end{array}\right]+\left[\begin{array}{ccc}
0 & 0 & 0 \\
0 & 0 & 0 \\
0 & 0 & 0 \\
0 & 0 & \mathrm{R}_{\mathrm{O}}
\end{array}\right]\left[\begin{array}{c}
\mathrm{V}_{\mathrm{g}} \\
\mathrm{I}_{\mathrm{g}} \\
\mathrm{I}_{\mathrm{O}}
\end{array}\right]
$$

Mode II: In this mode, $\mathrm{S}_{1}$ is OFF while $\mathrm{S}_{2}$ is $\mathrm{ON}$ as shown in Figure 2(c). During this interval, $\mathrm{C}_{1}$ and $\mathrm{L}_{1}$ are charged by the input voltage $\mathrm{V}_{\mathrm{g}}$ due to the series connection between them while $\mathrm{L}_{1}$ is isolated from the supply. $\mathrm{L}_{1}$ discharges to the load while the output filter absorbs the ripples.

$$
\begin{aligned}
& \dot{\mathrm{V}}_{\mathrm{C} 1}=\frac{\mathrm{I}_{\mathrm{L} 1}}{\mathrm{C}_{1}} \\
& \dot{\mathrm{V}}_{\mathrm{C} 2}=-\frac{\mathrm{I}_{\mathrm{L} 2}}{\mathrm{C}_{2}}-\frac{\mathrm{I}_{\mathrm{O}}}{\mathrm{C}_{2}} \\
& \dot{\mathrm{I}}_{\mathrm{L} 1}=-\frac{\mathrm{V}_{\mathrm{C} 1}}{\mathrm{~L}_{1}}-\left(\frac{\mathrm{R}_{1}+\mathrm{r}_{1}}{\mathrm{~L}_{1}}\right) \mathrm{I}_{\mathrm{L} 1}+\frac{\mathrm{V}_{\mathrm{g}}}{\mathrm{L}_{1}}
\end{aligned}
$$




$$
\dot{\mathrm{I}}_{\mathrm{L} 2}=-\frac{\mathrm{I}_{\mathrm{L} 2} \mathrm{r}_{2}}{\mathrm{~L}_{2}}+\frac{\mathrm{I}_{\mathrm{O}} \mathrm{R}_{\mathrm{O}}}{\mathrm{L}_{2}}
$$

Expressing in state space form $\dot{\mathrm{X}}_{\mathrm{i}}=\mathrm{A}_{\mathrm{i}} \mathrm{X}+\mathrm{B}_{\mathrm{i}} \mathrm{U}$ where $\mathrm{i}=2$ for mode 2 yields

$$
\dot{\mathrm{X}}_{2}=\left[\begin{array}{l}
\dot{\mathrm{V}}_{\mathrm{C} 1} \\
\dot{\mathrm{V}}_{\mathrm{C} 2} \\
\dot{\mathrm{I}}_{\mathrm{L} 1} \\
\dot{\mathrm{I}}_{\mathrm{L} 2}
\end{array}\right]=\left[\begin{array}{cccc}
0 & 0 & \frac{1}{\mathrm{C}_{1}} & 0 \\
0 & 0 & 0 & \frac{-1}{\mathrm{C}_{2}} \\
\frac{-1}{\mathrm{~L}_{1}} & 0 & \frac{-\left(\mathrm{R}_{1}+\mathrm{r}_{1}\right)}{\mathrm{L}_{1}} & 0 \\
0 & 0 & 0 & \frac{-\mathrm{r}_{2}}{\mathrm{~L}_{2}}
\end{array}\right]\left[\begin{array}{l}
\mathrm{V}_{\mathrm{C} 1} \\
\mathrm{~V}_{\mathrm{C} 2} \\
\mathrm{I}_{\mathrm{L} 1} \\
\mathrm{I}_{\mathrm{L} 2}
\end{array}\right]+\left[\begin{array}{ccc}
0 & 0 & 0 \\
0 & 0 & \frac{-1}{\mathrm{C}_{2}} \\
\frac{1}{\mathrm{~L}_{1}} & 0 & 0 \\
0 & 0 & \frac{\mathrm{R}_{\mathrm{O}}}{\mathrm{L}_{2}}
\end{array}\right]\left[\begin{array}{l}
\mathrm{V}_{\mathrm{g}} \\
\mathrm{I}_{\mathrm{g}} \\
\mathrm{I}_{\mathrm{O}}
\end{array}\right]
$$

The output equations for mode II are:

$$
\begin{aligned}
& \mathrm{V}_{\mathrm{C} 1}=\mathrm{V}_{\mathrm{C} 1} \\
& \mathrm{~V}_{\mathrm{C} 2}=\mathrm{V}_{\mathrm{C} 2} \\
& \mathrm{I}_{\mathrm{g}}=-\mathrm{I}_{\mathrm{L} 1} \\
& \mathrm{~V}_{\mathrm{O}}=\mathrm{I}_{\mathrm{O}} \mathrm{R}_{\mathrm{O}}
\end{aligned}
$$

Expressing the output in the form $Y_{i}=E_{i} X+F_{i} U$ where $\mathrm{i}=2$ for mode 2 yields

$$
\mathrm{Y}_{2}=\left[\begin{array}{c}
\mathrm{V}_{\mathrm{C} 1} \\
\mathrm{~V}_{\mathrm{C} 2} \\
\mathrm{I}_{\mathrm{g}} \\
\mathrm{V}_{\mathrm{O}}
\end{array}\right]=\left[\begin{array}{cccc}
1 & 0 & 0 & 0 \\
0 & 1 & 0 & 0 \\
0 & 0 & -1 & 0 \\
0 & 0 & 0 & 0
\end{array}\right]\left[\begin{array}{c}
\mathrm{V}_{\mathrm{C} 1} \\
\mathrm{~V}_{\mathrm{C} 2} \\
\mathrm{I}_{\mathrm{L} 1} \\
\mathrm{I}_{\mathrm{L} 2}
\end{array}\right]+\left[\begin{array}{ccc}
0 & 0 & 0 \\
0 & 0 & 0 \\
0 & 0 & 0 \\
0 & 0 & \mathrm{R}_{\mathrm{O}}
\end{array}\right]\left[\begin{array}{c}
\mathrm{V}_{\mathrm{g}} \\
\mathrm{I}_{\mathrm{g}} \\
\mathrm{I}_{\mathrm{O}}
\end{array}\right]
$$

The state equations are then averaged and expressed as

$$
\begin{aligned}
& \dot{X}=A X+B U \\
& Y=E X+F U
\end{aligned}
$$

Where $A=\sum_{i=1}^{n} A_{i} D_{i}, B=\sum_{i=1}^{n} B_{i} D_{i}, E=\sum_{i=1}^{n} E_{i} D_{i}, F=\sum_{i=1}^{n} F_{i} D_{i}, n$ is the number of switching states involved, $i=$ switched state and $D$ is the duty ratio of the switched state. For this circuit, $n=2$ since two switching states are involved (as in typical buck-boost converter), $\mathrm{D}_{1}=\mathrm{D}$ and $\mathrm{D}_{2}=\mathrm{D}^{\prime}=1-\mathrm{D}$ for modes I and II respectively. Therefore, $A=A_{1} D+A_{2}(1-D), \quad B=B_{1} D+B_{2}(1-D), \quad E=E_{1} D+E_{2}(1-D) \quad$ and $F=F_{1} D+F_{2}(1-D)$.

$$
\begin{aligned}
& \dot{\mathrm{X}}=\left[\begin{array}{l}
\dot{\mathrm{V}}_{\mathrm{C} 1} \\
\dot{\mathrm{V}}_{\mathrm{C} 2} \\
\dot{\mathrm{I}}_{\mathrm{L} 1} \\
\dot{\mathrm{I}}_{\mathrm{L} 2}
\end{array}\right]=\left[\begin{array}{cccc}
0 & 0 & \frac{1}{\mathrm{C}_{1}} & 0 \\
0 & 0 & \frac{-\mathrm{D}}{\mathrm{C}_{2}} & \frac{-(1-\mathrm{D})}{\mathrm{C}_{2}} \\
\frac{-1}{\mathrm{~L}_{1}} & 0 & \frac{-\left(\mathrm{R}_{1}+\mathrm{r}_{1}\right)}{\mathrm{L}_{1}} & \frac{0}{\mathrm{~L}_{1}} \\
0 & 0 & 0 & \frac{-\mathrm{r}_{2}}{\mathrm{~L}_{2}}
\end{array}\right]\left[\begin{array}{l}
\mathrm{V}_{\mathrm{C} 1} \\
\mathrm{~V}_{\mathrm{C} 2} \\
\mathrm{I}_{\mathrm{L} 1} \\
\mathrm{I}_{\mathrm{L} 2}
\end{array}\right]+\left[\begin{array}{ccc}
0 & 0 & 0 \\
0 & 0 & \frac{-1}{\mathrm{C}_{2}} \\
\frac{(1-\mathrm{D})}{\mathrm{L}_{1}} & 0 & \frac{\mathrm{DR}_{\mathrm{O}}}{\mathrm{L}_{1}} \\
\frac{\mathrm{D}}{\mathrm{L}_{2}} & 0 & \frac{\left(1-\mathrm{D}_{\mathrm{O}}\right.}{\mathrm{L}_{2}}
\end{array}\right]\left[\begin{array}{c}
\mathrm{V}_{\mathrm{g}} \\
\mathrm{I}_{\mathrm{g}} \\
\mathrm{I}_{\mathrm{O}}
\end{array}\right] \\
& \mathrm{Y}=\left[\begin{array}{c}
\mathrm{V}_{\mathrm{C} 1} \\
\mathrm{~V}_{\mathrm{C} 2} \\
\mathrm{I}_{\mathrm{g}} \\
\mathrm{V}_{\mathrm{O}}
\end{array}\right]=\left[\begin{array}{cccc}
1 & 0 & 0 & 0 \\
0 & 1 & 0 & 0 \\
0 & 0 & -(1-\mathrm{D}) & -\mathrm{D} \\
0 & 0 & 0 & 0
\end{array}\right]\left[\begin{array}{c}
\mathrm{V}_{\mathrm{C} 1} \\
\mathrm{~V}_{\mathrm{C} 2} \\
\mathrm{I}_{\mathrm{L} 1} \\
\mathrm{I}_{\mathrm{L} 2}
\end{array}\right]+\left[\begin{array}{ccc}
0 & 0 & 0 \\
0 & 0 & 0 \\
0 & 0 & 0 \\
0 & 0 & \mathrm{R}_{\mathrm{O}}
\end{array}\right]\left[\begin{array}{l}
\mathrm{V}_{\mathrm{g}} \\
\mathrm{I}_{\mathrm{g}} \\
\mathrm{I}_{\mathrm{O}}
\end{array}\right]
\end{aligned}
$$

(31) And (32) are the modelled averaged steady-state equations of the circuit. The choice of $V_{O}$ and $I_{O}$ as output and input respectively resulted in the feedforward matrices in (18), (28) and (32) nonzero. If $\mathrm{V}_{\mathrm{O}}$ is not considered as output, all these feedforward matrices will be zero. However, the choice of Ig as both output and input didn't 
affect the feedforward matrices nor any input matrix because the system's steady-state response is independent of the input $\mathrm{I}_{\mathrm{g}}$ but $\mathrm{V}_{\mathrm{g}}$ and $\mathrm{I}_{\mathrm{O}}$. This is important in controller design.

\section{SMALL SIGNAL ANALYSIS}

Small signal perturbations $\tilde{d}(t), \tilde{v}_{g}(t), \tilde{i}_{g}(t)$ and $\tilde{i}_{O}(t)$ are applied to the steady-state duty ratio $(D)$ and input variables $\left(V_{g}, I_{g}\right.$, and $\left.I_{O}\right)$ respectively to obtain the small signal model. These perturbations cause the dynamic state variables $\mathrm{v}_{\mathrm{C} 1}, \mathrm{v}_{\mathrm{C} 2}, \mathrm{i}_{\mathrm{L} 1}$ and $\mathrm{i}_{\mathrm{L} 2}$ to vary by $\tilde{\mathrm{v}}_{\mathrm{C} 1}, \tilde{\mathrm{v}}_{\mathrm{C} 2}, \tilde{\mathrm{I}}_{\mathrm{L} 1}$ and $\tilde{\mathrm{I}}_{\mathrm{L} 2}$ respectively [56]. The relationship between a dynamic variable $\mathrm{x}$, its steady state value $\mathrm{X}$ and perturbation $\tilde{\mathrm{X}}$ is given as

$$
\mathrm{x}=\mathrm{X}+\tilde{\mathrm{x}}
$$

Differentiating (33) with respect to time yields

$$
\dot{\mathrm{X}}=\dot{\mathrm{X}}+\dot{\tilde{\mathrm{X}}}
$$

Steady-state variables in (29) are substituted with dynamic variables for small signal analysis to yield (35)

$$
\dot{\mathrm{x}}=\left(\mathrm{A}_{1} \mathrm{~d}+\mathrm{A}_{2}(1-\mathrm{d})\right) \mathrm{x}+\left(\mathrm{B}_{1} \mathrm{~d}+\mathrm{B}_{2}(1-\mathrm{d})\right) \mathrm{u}
$$

Substituting (33) and (34) into (35), neglecting products of two small signal perturbations and rearranging yields

$$
\dot{\mathrm{X}}+\dot{\tilde{\mathrm{x}}}=\mathrm{AX}+\mathrm{BU}+\mathrm{A} \tilde{\mathrm{x}}+\mathrm{B} \tilde{\mathrm{u}}+\left[\left(\mathrm{A}_{1}-\mathrm{A}_{2}\right) \mathrm{X}+\left(\mathrm{B}_{1}-\mathrm{B}_{2}\right) \mathrm{U}\right] \tilde{\mathrm{d}}
$$

(36) Is the generalised large signal state equation for a model. Matching steady state and perturbation terms together shows

$$
\begin{aligned}
& \dot{X}=A X+B U=0 \\
& \dot{\tilde{x}}=A \tilde{x}+B \tilde{u}+\left[\left(A_{1}-A_{2}\right) X+\left(B_{1}-B_{2}\right) U\right] \tilde{\mathrm{d}}
\end{aligned}
$$

(37) $=0$ because derivative of a constant (steady state) $\dot{X}=0$. (37) Is the generalised steady state model while (38) is generalised small signal model.

Simplifying (36) further yields

$$
\mathrm{X}=-\mathrm{BUA}^{-1}
$$

Similarly, for the steady state output $\mathrm{Y}=\mathrm{EX}+\mathrm{FU}$, its dynamic signal after small signal analysis is given as

$$
y=Y+\tilde{y}=E X+F U+E \tilde{x}+F \tilde{u}+\left[\left(E_{1}-E_{2}\right) X+\left(F_{1}-F_{2}\right) U\right] \tilde{d}
$$

(40) Is the generalized large signal output equation for a model. Matching steady state and perturbation terms together shows

$$
\begin{aligned}
& Y=E X+F U \\
& \tilde{y}=E \tilde{x}+F \tilde{u}+\left[\left(E_{1}-E_{2}\right) X+\left(F_{1}-F_{2}\right) U\right] \tilde{d}
\end{aligned}
$$

(41) Is the generalized steady state output equation while (42) is the generalised small signal equation. Substituting (39) into (41) yields

$$
\begin{aligned}
& Y=-E_{B U A^{-1}+F U} \\
& Y=\left(F-E B A^{-1}\right) U
\end{aligned}
$$


Evaluating and simplifying (38) yields (45) to (48)

$$
\begin{aligned}
& \dot{\tilde{\mathrm{v}}}_{\mathrm{C} 1}=\frac{\tilde{\mathrm{L}}_{\mathrm{L} 1}}{\mathrm{C}_{1}} \\
& \dot{\tilde{\mathrm{v}}}_{\mathrm{C} 2}=\frac{-\mathrm{D} \tilde{\mathrm{l}}_{\mathrm{L} 1}-(1-\mathrm{D}) \tilde{\mathrm{I}}_{\mathrm{L} 2}-\tilde{\mathrm{i}}_{0}+\left(\mathrm{I}_{\mathrm{L} 2}-\mathrm{I}_{\mathrm{L} 1}\right) \tilde{\mathrm{d}}}{\mathrm{C}_{2}} \\
& \dot{\tilde{\mathrm{I}}}_{\mathrm{L} 1}=\frac{-\tilde{\mathrm{v}}_{\mathrm{C} 1}-\left(\mathrm{R}_{1}+\mathrm{r}_{1}\right) \tilde{\mathrm{l}}_{\mathrm{L} 1}+(1-\mathrm{D}) \tilde{\mathrm{v}}_{\mathrm{g}}+\left(D \mathrm{R}_{0}\right) \tilde{\mathrm{I}}_{0}+\left(\mathrm{I}_{0} \mathrm{R}_{0}-\mathrm{V}_{\mathrm{g}}\right) \tilde{\mathrm{d}}}{\mathrm{L}_{1}} \\
& \dot{\tilde{\mathrm{I}}}_{\mathrm{L} 2}=\frac{-\mathrm{r}_{2} \tilde{\mathrm{l}}_{\mathrm{L} 2}+\mathrm{D} \tilde{\mathrm{v}}_{\mathrm{g}}+(1-\mathrm{D}) \mathrm{R}_{0} \tilde{\mathrm{I}}_{0}+\left(\mathrm{V}_{\mathrm{g}}-\mathrm{I}_{0} \mathrm{R}_{0}\right) \tilde{\mathrm{d}}}{\mathrm{L}_{2}}
\end{aligned}
$$

Taking Laplace transform and simplification yields

$$
\begin{aligned}
& \mathrm{sC}_{1} \tilde{\mathrm{v}}_{\mathrm{c} 1}(\mathrm{~s})=\tilde{\mathrm{l}}_{\mathrm{L} 1}(\mathrm{~s}) \\
& \mathrm{sC}_{2} \tilde{\mathrm{v}}_{\mathrm{c} 2}(\mathrm{~s})=-\mathrm{D} \tilde{\mathrm{l}}_{\mathrm{L} 1}(\mathrm{~s})-(1-\mathrm{D}) \tilde{\mathrm{l}}_{\mathrm{L} 2}(\mathrm{~s})-\tilde{\mathrm{l}}_{0}(\mathrm{~s})+\left(\mathrm{I}_{\mathrm{L} 2}-\mathrm{I}_{\mathrm{L} 1}\right) \tilde{\mathrm{d}}(\mathrm{s}) \\
& \left(\mathrm{sL}_{1}+\mathrm{R}_{1}+\mathrm{r}_{1}\right) \tilde{\mathrm{l}}_{\mathrm{L} 1}(\mathrm{~s})=-\tilde{\mathrm{v}}_{\mathrm{C} 1}(\mathrm{~s})+(1-\mathrm{D}) \tilde{\mathrm{v}}_{\mathrm{g}}(\mathrm{s})+\left(\mathrm{DR}_{0}\right) \tilde{\mathrm{i}}_{0}(\mathrm{~s})+\left(\mathrm{I}_{0} \mathrm{R}_{0}-\mathrm{V}_{\mathrm{g}}\right) \tilde{\mathrm{d}}(\mathrm{s}) \\
& \left(\mathrm{sL}_{2}+\mathrm{r}_{2}\right) \tilde{\mathrm{l}}_{\mathrm{L} 2}(\mathrm{~s})=\tilde{\mathrm{l}}_{\mathrm{L} 2}(\mathrm{~s})+\mathrm{D} \tilde{\mathrm{v}}_{\mathrm{g}}(\mathrm{s})+\mathrm{R}_{0}(1-\mathrm{D}) \tilde{\mathrm{l}}_{0}(\mathrm{~s})+\left(\mathrm{V}_{\mathrm{g}}-\mathrm{I}_{0} \mathrm{R}_{0}\right) \tilde{\mathrm{d}}(\mathrm{s})
\end{aligned}
$$

Further simplification and substitutions yields

$$
\begin{aligned}
& \tilde{\mathrm{v}}_{\mathrm{c} 1}(\mathrm{~s})=\frac{(1-\mathrm{D}) \tilde{\mathrm{v}}_{\mathrm{g}}(\mathrm{s})+\left(\mathrm{DR}_{0}\right) \tilde{\mathrm{I}}_{0}(\mathrm{~s})+\left(\mathrm{I}_{0} \mathrm{R}_{0}-\mathrm{V}_{\mathrm{g}}\right) \tilde{\mathrm{d}}(\mathrm{s})}{\left(\mathrm{sL}_{1}+\mathrm{R}\right)\left(\mathrm{sC}_{1}+1\right)} \\
& \tilde{\mathrm{v}}_{\mathrm{c} 2}(\mathrm{~s})=-\frac{\mathrm{s}^{2}\left(\mathrm{~L}_{1}+\mathrm{L}_{2}\right)+\mathrm{s}\left(\mathrm{R}+\mathrm{r}_{2}\right)+\frac{1}{\mathrm{C}_{1}}}{\left(\mathrm{~s}^{2} \mathrm{C}_{1} \mathrm{~L}_{1}+\mathrm{sC}_{1} \mathrm{R}+1\right)\left(\mathrm{sL}_{2}+\mathrm{r}_{2}\right) \mathrm{s} \frac{\mathrm{C}_{2}}{\mathrm{C}_{1}}} \mathrm{DD}^{\prime} \tilde{\mathrm{v}}_{\mathrm{g}} \\
& -\frac{s^{3} L_{1} L_{2}+s^{2}\left(L_{2}\left(D^{2} R_{0}+R\right)+L_{1}\left(R_{0} D^{\prime 2}+r_{2}\right)\right)+s\left(R_{0} D^{\prime 2} R+r_{2}\left(R+D^{2} R_{0}\right)+\frac{L_{2}}{C_{1}}\right)+\frac{R_{0} D^{\prime 2}+r_{2}}{C_{1}}}{\left(s^{2} C_{1} L_{1}+s C_{1} R+1\right)\left(s L_{2}+r_{2}\right) s \frac{C_{2}}{C_{1}}} \tilde{i}_{0}(s) \\
& +\frac{s^{3} L_{1} L_{2} I+s^{2}\left(L_{1} r_{2} I+L_{2} R I+D L_{2} V-D^{\prime} L_{1} V\right)+s\left(R r_{2} I+\frac{L_{2}}{C_{1}} I+D r_{2} V+D r_{2}-D^{\prime} R V\right)+\frac{r_{2} I-D^{\prime} V}{C_{1}}}{\left(s^{2} C_{1} L_{1}+s C_{1} R+1\right)\left(s L_{2}+r_{2}\right) s \frac{C_{2}}{C_{1}}} \tilde{d}(s) \\
& \tilde{\mathrm{l}}_{\mathrm{L} 1}(\mathrm{~s})=\frac{\mathrm{SC}_{1}(1-\mathrm{D}) \tilde{\mathrm{v}}_{\mathrm{g}}(\mathrm{s})+\mathrm{SC}_{1} \mathrm{DR}_{0} \tilde{\mathrm{I}}_{0}(\mathrm{~s})+\mathrm{SC}_{1}\left(\mathrm{I}_{0} \mathrm{R}_{0}-\mathrm{V}_{\mathrm{g}}\right) \tilde{\mathrm{d}}(\mathrm{s})}{\mathrm{s}^{2} \mathrm{C}_{1} \mathrm{~L}_{1}+\mathrm{sC} \mathrm{C}_{1} \mathrm{R}+1} \\
& \tilde{\mathrm{l}}_{\mathrm{L} 2}(\mathrm{~s})=\frac{\mathrm{D} \tilde{\mathrm{v}}_{\mathrm{g}}(\mathrm{s})+\mathrm{R}_{0}(1-\mathrm{D}) \tilde{\mathrm{I}}_{0}(\mathrm{~s})+\left(\mathrm{V}_{\mathrm{g}}-\mathrm{I}_{0} \mathrm{R}_{0}\right) \tilde{\mathrm{d}}(\mathrm{s})}{\mathrm{sL}_{2}+\mathrm{r}_{2}}
\end{aligned}
$$

where $R=R_{1}+r_{1}, V=V_{g}-V_{0}, D^{\prime}=1-D$ and $I=I_{L 2}-I_{L 1}$.

The small signal equations of the states $\tilde{v}_{\mathrm{c} 1}(\mathrm{~s})$ and $\tilde{\mathrm{v}}_{\mathrm{c} 2}(\mathrm{~s})$ as shown in (53) and (54) are not identical, likewise $\tilde{\mathrm{l}}_{\mathrm{L} 1}(\mathrm{~s})$ and $\tilde{\mathrm{l}}_{\mathrm{L} 2}(\mathrm{~s})$ as shown in (55) and (56) are also non identical. An explanation to this non-identicality is due to the asymmetry of this topology. This asymmetry is explained by the difference in the gain curves obtained when taking the output across $C_{1}$ as done in [13] and when taken across $C_{2}$ as done in this presentation. The gain of the two variant topologies shows that for any given operational parameters, $\mathrm{V}_{\mathrm{C} 1} \neq \mathrm{V}_{\mathrm{C} 2}$. The models presented in [41], [44] have the above-mentioned states to be identical because inverters were considered and not DC-DC converter thus the topologies are entirely different. However, the poles of $\tilde{1}_{\mathrm{L} 1}(\mathrm{~s})$ and $\tilde{\mathrm{l}}_{\mathrm{L} 2}(\mathrm{~s})$ are contained in the poles of $\widetilde{\mathrm{V}}_{\mathrm{C} 2}(\mathrm{~s})$ thus (55) and (56) could be re-written as 


$$
\begin{gathered}
\tilde{\mathrm{I}}_{\mathrm{L} 1}(\mathrm{~s})=\frac{\mathrm{SC}_{1}(1-\mathrm{D}) \tilde{\mathrm{v}}_{\mathrm{g}}(\mathrm{s})+\mathrm{SC}_{1} \mathrm{DR}_{0} \tilde{\mathrm{I}}_{0}(\mathrm{~s})+\mathrm{SC}_{1}\left(\mathrm{I}_{0} \mathrm{R}_{0}-\mathrm{V}_{\mathrm{g}}\right) \tilde{\mathrm{d}}(\mathrm{s})}{\left(\mathrm{s}^{2} \mathrm{C}_{1} \mathrm{~L}_{1}+\mathrm{sC}_{1} \mathrm{R}+1\right)\left(\mathrm{sL}_{2}+\mathrm{r}_{2}\right) \mathrm{s} \frac{\mathrm{C}_{2}}{\mathrm{C}_{1}}}\left(\mathrm{~s}_{2}+\mathrm{r}_{2}\right) \mathrm{s} \frac{\mathrm{C}_{2}}{\mathrm{C}_{1}} \\
\tilde{\mathrm{I}}_{\mathrm{L} 2}(\mathrm{~s})=\frac{\mathrm{D} \tilde{\mathrm{v}}_{\mathrm{g}}(\mathrm{s})+\mathrm{R}_{0}(1-\mathrm{D}) \tilde{\mathrm{I}}_{0}(\mathrm{~s})+\left(\mathrm{V}_{\mathrm{g}}-\mathrm{I}_{0} \mathrm{R}_{0}\right) \tilde{\mathrm{d}}(\mathrm{s})}{\left(\mathrm{s}^{2} \mathrm{C}_{1} \mathrm{~L}_{1}+\mathrm{sC}_{1} \mathrm{R}+1\right)\left(\mathrm{sL}_{2}+\mathrm{r}_{2}\right) \mathrm{s} \frac{\mathrm{C}_{2}}{\mathrm{C}_{1}}}\left(\mathrm{~s}^{2} \mathrm{C}_{1} \mathrm{~L}_{1}+\mathrm{sC}_{1} \mathrm{R}+1\right) \mathrm{s} \frac{\mathrm{C}_{2}}{\mathrm{C}_{1}}
\end{gathered}
$$

\subsection{Transfer functions}

The small signal models presented in (53) to (56) were used to obtain the transfer functions $\left(G_{\text {input }}^{\text {state }}\right)$ between state variable and system input. This was done by considering one system input at a time and assuming other system inputs to be zero [41], [44], [48].

$$
\begin{aligned}
G_{\tilde{v}_{g}}^{\tilde{v}_{C 1}} & =\frac{(1-D)}{\left(s L_{1}+R\right)\left(s C_{1}+1\right)} \\
\mathrm{G}_{\mathrm{i}_{\mathrm{i}}}^{\widetilde{\mathrm{C}_{1}}} & =\frac{\mathrm{DR}_{0}}{\left(\mathrm{sL}_{1}+R\right)\left(\mathrm{sC}_{1}+1\right)} \\
\mathrm{G}_{\widetilde{\mathrm{d}}}^{\widetilde{\mathrm{V}} \mathrm{C} 1} & =\frac{\left(\mathrm{I}_{0} \mathrm{R}_{0}-\mathrm{V}_{\mathrm{g}}\right)}{\left(\mathrm{sL}_{1}+R\right)\left(\mathrm{sC}_{1}+1\right)} \\
\mathrm{G}_{\widetilde{\mathrm{v}}}^{\widetilde{\mathrm{V}} \mathrm{c} 2} & =-\frac{\left[\mathrm{s}^{2}\left(\mathrm{~L}_{1}+\mathrm{L}_{2}\right)+\mathrm{s}\left(\mathrm{R}+\mathrm{r}_{2}\right)+\frac{1}{\mathrm{C}_{1}}\right] \mathrm{DD}^{\prime}}{\left(\mathrm{s}^{2} \mathrm{C}_{1} \mathrm{~L}_{1}+\mathrm{sC}_{1} \mathrm{R}+1\right)\left(\mathrm{sL}_{2}+\mathrm{r}_{2}\right) \mathrm{s} \frac{\mathrm{C}_{2}}{\mathrm{C}_{1}}}
\end{aligned}
$$

$=-\frac{s^{3}+s^{2}\left(\frac{\left(L_{2}\left(D^{2} R_{0}+R\right)+L_{1}\left(R_{0} D^{\prime 2}+r_{2}\right)\right.}{\mathrm{V}_{1} \mathrm{~L}_{2}}\right)+s\left(\frac{\mathrm{R}_{0} \mathrm{C}_{1} \mathrm{D}^{\prime 2} \mathrm{R}+\mathrm{r}_{2} \mathrm{C}_{1}\left(\mathrm{R}+\mathrm{D}^{2} \mathrm{R}_{0}\right)+\mathrm{L}_{2}}{\mathrm{~L}_{1} \mathrm{~L}_{2} \mathrm{C}_{1}}\right)+\frac{\mathrm{R}_{0} \mathrm{D}^{\prime 2}+\mathrm{r}_{2}}{\mathrm{~L}_{1} \mathrm{~L}_{2} \mathrm{C}_{1}}}{\left(\mathrm{~s}^{2} \mathrm{C}_{1} \mathrm{~L}_{1}+\mathrm{sC}_{1} \mathrm{R}+1\right)\left(\mathrm{sL}_{2}+\mathrm{r}_{2}\right) \mathrm{s} \frac{\mathrm{C}_{2}}{\mathrm{C}_{1}}}$

$G_{\widetilde{\mathrm{d}}}^{\widetilde{V}_{C 2}}$

$$
=\frac{s^{3} L_{1} L_{2} I+s^{2}\left(L_{1} r_{2} I+L_{2} R I+D L_{2} V-D^{\prime} L_{1} V\right)+s\left(R_{2} I+\frac{L_{2}}{C_{1}} I+D r_{2} V+D r_{2}-D^{\prime} R V\right)+\frac{r_{2} I-D^{\prime} V}{C_{1}}}{\left(s^{2} C_{1} L_{1}+s C_{1} R+1\right)\left(s L_{2}+r_{2}\right) s \frac{C_{2}}{C_{1}}}
$$

$$
\begin{aligned}
& G_{\widetilde{\mathrm{v}}_{\mathrm{g}}}^{\tilde{\mathrm{L}}_{1}}=\frac{\mathrm{SC}_{1}(1-\mathrm{D})}{\mathrm{S}^{2} \mathrm{C}_{1} \mathrm{~L}_{1}+\mathrm{SC}_{1} \mathrm{R}+1} \\
& \mathrm{G}_{\tilde{\mathrm{I}}_{0}}^{\tilde{\mathrm{L}}_{1}}=\frac{\mathrm{SC}_{1} \mathrm{DR}_{0}}{\mathrm{~s}^{2} \mathrm{C}_{1} \mathrm{~L}_{1}+\mathrm{sC}_{1} \mathrm{R}+1} \\
& G_{\tilde{d}}^{\tilde{L}_{1}}=\frac{S^{2} C_{1}\left(I_{0} R_{0}-V_{g}\right) \tilde{d}}{S^{2} C_{1} L_{1}+s C_{1} R+1} \\
& \mathrm{G}_{\widetilde{\mathrm{v}}_{\mathrm{g}}}^{\tilde{\mathrm{l}}_{2}}=\frac{\mathrm{D}}{\mathrm{sL}_{2}+\mathrm{r}_{2}} \\
& G_{\tilde{\mathbf{I}}_{0}}^{\tilde{I}_{L 2}}=\frac{R_{0}(1-D)}{s L_{2}+r_{2}} \\
& G_{\tilde{\mathrm{d}}}^{\tilde{\mathrm{I}}_{2}}=\frac{\left(\mathrm{V}_{\mathrm{g}}-\mathrm{I}_{0} \mathrm{R}_{0}\right) \tilde{\mathrm{d}}(\mathrm{s})}{\mathrm{sL}_{2}+\mathrm{r}_{2}}
\end{aligned}
$$




\section{ANALYSIS}

The poles and zeros of the transfer functions are discussed in this section. Roots of functions not greater than degree 2 are fully discussed while those of degree 3 and 4 are just introduced due to the complexity involved. Pole-zero maps have been used to analyse dynamic models of dc-ac ISCs [41], [44], [45], [48], [49], [51], analytical method is used here for analyses due to the asymmetry of this topology which resulted in (62) having so many variables.

a. Starting with the first transfer function $G_{\widetilde{V}_{g}}^{\widetilde{V}_{C 1}}$, together with $G_{\tilde{1}_{0}}^{\widetilde{V}_{C 1}}$ and $G_{\widetilde{d}} \widetilde{V}_{C 1}$, they have two poles all negative located at $\mathrm{s}=-\frac{\mathrm{R}_{1}+\mathrm{r}_{1}}{\mathrm{~L}_{1}}$ and $\mathrm{s}=\frac{-1}{\mathrm{C}_{1}}$. They all have no zero.

b. $\mathrm{G}_{\widetilde{\mathrm{V}}_{\mathrm{g}}}^{\widetilde{\mathrm{V}}_{\mathrm{C} 2}}$ together with $\mathrm{G}_{\tilde{\mathrm{I}}_{0}}^{\widetilde{V}_{\mathrm{C} 2}}$ and $\mathrm{G}_{\widetilde{\mathrm{d}}}^{\widetilde{\mathrm{V}}_{\mathrm{C} 2}}$, they have four poles all non-positive located at $0,-\frac{\mathrm{r}_{2}}{\mathrm{~L}_{2}}$ and $-\frac{R}{2 \mathrm{~L}_{1}} \pm$ $\frac{\sqrt{\left(\frac{\mathrm{R}}{\mathrm{L}_{1}}\right)^{2}-\left(\frac{4}{\mathrm{C}_{1} \mathrm{~L}_{1}}\right)}}{2}$. The pole $-\frac{\mathrm{R}}{2 \mathrm{~L}_{1}}+\frac{\sqrt{\left(\frac{R}{\mathrm{~L}_{1}}\right)^{2}-\left(\frac{4}{\mathrm{C}_{1} \mathrm{~L}_{1}}\right)}}{2}$ is also non positive because $\frac{\left(\frac{R}{\mathrm{~L}_{1}}\right)^{2}-4\left(\frac{1}{\mathrm{C}_{1} \mathrm{~L}_{1}}\right)}{4} \ngtr\left(\frac{\mathrm{R}}{2 \mathrm{~L}_{1}}\right)^{2} \Rightarrow \frac{-1}{\mathrm{C}_{1} \mathrm{~L}_{1}} \ngtr 0$ since $\mathrm{C}_{1}$ and $\mathrm{L}_{1}$ are all positive. Provided $\left(\frac{R}{\mathrm{~L}_{1}}\right)^{2} \geq 4\left(\frac{1}{\mathrm{C}_{1} \mathrm{~L}_{1}}\right)$, or simply $\mathrm{L}_{1} \leq \frac{\mathrm{C}_{1}}{4} \mathrm{R}^{2}$, it has all real non-positive poles. It has two zeros at $-\frac{R+\mathrm{r}_{2}}{2\left(\mathrm{~L}_{1}+\mathrm{L}_{2}\right)} \pm \sqrt{\left(\frac{\mathrm{R}+\mathrm{r}_{2}}{2\left(\mathrm{~L}_{1}+\mathrm{L}_{2}\right)}\right)^{2}-\left(\frac{1}{\mathrm{C}_{1}\left(\mathrm{~L}_{1}+\mathrm{L}_{2}\right)}\right)}$ all the zeros are negative because $\left(\frac{R+\mathrm{r}_{2}}{2\left(\mathrm{~L}_{1}+\mathrm{L}_{2}\right)}\right)^{2}-\left(\frac{1}{\mathrm{C}_{1}\left(\mathrm{~L}_{1}+\mathrm{L}_{2}\right)}\right) \geq\left(\frac{\mathrm{R}+\mathrm{r}_{2}}{2\left(\mathrm{~L}_{1}+\mathrm{L}_{2}\right)}\right)^{2}$ since $\frac{-1}{\mathrm{C}_{1}\left(\mathrm{~L}_{1}+\mathrm{L}_{2}\right)} \geq 0$ due to the fact that both $\mathrm{C}_{1}, \mathrm{~L}_{1}$ and $\mathrm{L}_{2}$ are all positive.

c. $G_{\tilde{I}_{O}}^{\widetilde{V}_{\mathrm{C} 2}}$ has three zeros and their locations can be analysed based on the given operating conditions because the polynomial being of degree three and with so many parameters makes it difficult to present a generalised analysis.

d. $G_{\widetilde{d}}^{\widetilde{V}} \widetilde{C}_{2}$ has three zeros and are given by the roots of the polynomial $s^{3} L_{1} L_{2} I+s^{2}\left(L_{1} r_{2} I+L_{2} R I+D L_{2} V-\right.$ $\left.D^{\prime} L_{1} V\right)+s\left(R_{2} I+\frac{L_{2}}{C_{1}} I+D r_{2} V+D r_{2}-D^{\prime} R V\right)+\frac{r_{2} I-D^{\prime} V}{C_{1}}$. Analysing the behaviour of all the possible roots of this cubic polynomial analytically is complex and involves so much mathematics beyond the scope of this paper because I and $\mathrm{V}$ are variables whose values vary for different operating points. This is evident as [41], [44] also analysed their quadratic $\mathrm{G}_{\tilde{d}}^{\widetilde{V}} \mathrm{C}$ by considering the parameters of a given circuit under given conditions. However, limited cases will be considered such as

$\mathrm{I}_{\mathrm{L} 1}=\mathrm{I}_{\mathrm{L} 2}$. If $\mathrm{I}_{\mathrm{L} 1}=\mathrm{I}_{\mathrm{L} 2}$, the polynomial reduces to degree two as

$s^{2}+s\left(\frac{D_{2}+\frac{D r_{2}}{V}-D^{\prime} R}{D L_{2}-D^{\prime} L_{1}}\right)-\frac{D^{\prime}}{\left(D L_{2}-D^{\prime} L_{1}\right) C_{1}}$. Its roots are given by

$-\frac{\mathrm{Dr}_{2}+\frac{\mathrm{Dr}_{2}}{\mathrm{~V}}-\mathrm{D}^{\prime} \mathrm{R}}{2\left(\mathrm{DL}_{2}-\mathrm{D}^{\prime} \mathrm{L}_{1}\right)} \pm \sqrt{\left(\frac{\mathrm{Dr}_{2}+\frac{\mathrm{Dr}_{2}}{\mathrm{~V}}-\mathrm{D}^{\prime} \mathrm{R}}{2\left(\mathrm{DL}_{2}-\mathrm{D}^{\prime} \mathrm{L}_{1}\right)}\right)^{2}-\frac{\mathrm{D}^{\prime}}{\left(\mathrm{DL}_{2}-\mathrm{D}^{\prime} \mathrm{L}_{1}\right) \mathrm{C}_{1}}}$.

$\mathrm{I}_{\mathrm{L} 1}=\mathrm{I}_{\mathrm{L} 2}$ and $\mathrm{V}_{\mathrm{g}}=\mathrm{V}_{\mathrm{O}}$, the equation reduces to $\mathrm{sDr}_{2}$ thus the zero exist at origin $(\mathrm{s}=0)$.

As shown by these two cases, the nature of the zeros varies for different points. An important point to note is that right-hand plane (RHP) zero may exist outside the conditions of case II. The existence of this RHP zero was also noticed in ZSI and q-ZSI which implies control limitations and high gain instability [41], [44], [45], [48], [49] thereby destabilizing the feedback loop.

e. $G_{\widetilde{v}_{g}}^{\tilde{L}_{L}}, G_{\tilde{I}_{O}}^{\tilde{I}_{L} 1}$ and $G_{\widetilde{d}}^{\tilde{L}_{L 1}}$ have all negative poles located at $-\frac{R}{2 \mathrm{~L}_{1}} \pm \frac{\sqrt{\left(\frac{\mathrm{R}}{\mathrm{L}_{1}}\right)^{2}-\left(\frac{4}{\mathrm{C}_{1} \mathrm{~L}_{1}}\right)}}{2}$. The poles are all negative because $\left(\frac{R}{\mathrm{~L}_{1}}\right)^{2}-\left(\frac{4}{\mathrm{C}_{1} \mathrm{~L}_{1}}\right) \geq\left(\frac{\mathrm{R}}{\mathrm{L}_{1}}\right)^{2}$ since $\frac{-4}{\mathrm{C}_{1} \mathrm{~L}_{1}} \geq 0$. The poles are real provided $\mathrm{L}_{1} \leq \mathrm{C}_{1}\left(\frac{R}{2}\right)^{2}$. They all have single zero and is located at $\mathrm{s}=0$.

f. $\quad G_{\widetilde{v}_{\mathrm{g}}}^{\tilde{L}_{L}}, G_{\tilde{1}_{O}}^{\tilde{I}_{L 2}}$ and $G_{\widetilde{\mathrm{d}}}^{\tilde{l}_{L 2}}$ have a single pole and no zero. The pole is located at $\mathrm{s}=-\frac{\mathrm{r}_{2}}{\mathrm{~L}_{2}}$.

From the above analysis, it can be deduced that the transfer functions $G_{\widetilde{v} g}^{\widetilde{v}}, G_{\tilde{1}_{0}}^{\widetilde{v}}$ and $G_{\widetilde{d}}^{\widetilde{v}} \mathrm{C}$ derived from the state $\tilde{\mathrm{v}}_{\mathrm{c} 1}(\mathrm{~s})$ and $\mathrm{G}_{\widetilde{\mathrm{v}}_{\mathrm{g}}}^{\tilde{\mathrm{L}}_{\mathrm{L} 2}}, \mathrm{G}_{\tilde{\mathrm{I}}_{\mathrm{O}}}^{\tilde{I}_{\mathrm{L} 2}}$ and $\mathrm{G}_{\widetilde{\mathrm{d}}}^{\tilde{I}_{\mathrm{L} 2}}$ derived from the state $\tilde{\mathrm{l}}_{\mathrm{L} 2}(\mathrm{~s})$ are generally stable regardless of parameter values. All their poles are negative-real and have no zeros. Smaller $L_{1}$ and $C_{1}$ increase the stability of the transfer functions $G_{\widetilde{v}_{g}}^{\widetilde{V}_{C 1}}, G_{\tilde{1}_{O}}^{\widetilde{v}_{C 1}}$ and $G_{\widetilde{d}}^{\widetilde{v}_{C 1}}$ by pushing their poles away from origin. Also, smaller $L_{2}$ will increase the system stability due to $G_{\widetilde{v}_{g}}^{\tilde{L}_{L}}, G_{\tilde{I}_{O}}^{\tilde{I}_{L 2}}$ and $G_{\widetilde{d}}^{\tilde{L}_{L 2}}$ by pushing their poles further away from the origin. Smaller values of $\mathrm{L}_{2}$ rather than larger values of $r_{2}$ are preferred because $r_{2}$ being a parasitic resistance will increase non-ideality such as parasitic voltage drop thereby reducing efficiency. 
The transfer functions $G_{\widetilde{v}_{g}}^{\tilde{L}_{L 1}}, G_{\tilde{I}_{0}}^{\tilde{L}_{L 1}}$ and $G_{\tilde{\mathrm{d}}}^{\tilde{I}_{\mathrm{L} 1}}$ derived from the state $\tilde{\mathrm{I}}_{\mathrm{L} 1}(\mathrm{~s})$ have marginal gain stability due to zero at the origin which implies control limitation [44]. Although all their poles are all negative, oscillations may occur if $\mathrm{L}_{1}>\mathrm{C}_{1}\left(\frac{R}{2}\right)^{2}$ due to the existence of a complex conjugate pair, else, the poles are negative and real with a smaller value of $\mathrm{L}_{1}$ pushing them further away from the origin.

It is now clear that the transfer functions $G_{\widetilde{v}_{\mathrm{g}}}^{\widetilde{v}_{\mathrm{C}}}, \mathrm{G}_{\tilde{1}_{\mathrm{O}}}^{\widetilde{v}_{\mathrm{C}}}$ and $\mathrm{G}_{\widetilde{\mathrm{d}}}^{\widetilde{v}_{\mathrm{C} 2}}$ derived from $\widetilde{\mathrm{v}}_{\mathrm{c} 2}(\mathrm{~s})$ are the most crucial because they indicate marginal stability due to the existence of a pole at origin and oscillation may occur if $L_{1}>$ $\frac{\mathrm{C}_{1}}{4} R^{2}$ due to the existence of complex conjugate pole pair. The zeros of $\mathrm{G}_{\widetilde{v}_{\mathrm{g}}}^{\widetilde{v}_{\mathrm{C} 2}}$ are all negative. From all the above analysis, it shows that the possibility of positive roots only exists in the zeros of $G_{\tilde{1}_{0}}^{\widetilde{v}_{C 2}}$ and $G_{\widetilde{d}}^{\widetilde{v}_{C 2}}$ which signifies control limitation and high gain instability and also exists in the ZSI and q-ZSI. This shows that the ZSI, q-ZSI and this DC-DC q-ZSC are non-minimum phase systems [48].

\section{VERIFICATION}

To verify these findings, operations of two converters were compared by simulating their performance on input voltage $\mathrm{V}_{\mathrm{g}}=12 \mathrm{~V}$, duty ratio $\mathrm{D}=0.63$ and $7 \Omega$ load using MATLAB SIMULINK. On one side was a converter based on arbitrary symmetric components as $C_{1}=C_{2}=400 \mu F, L_{1}=L_{2}=500 \mu H, R_{1}=R_{2}=$ $0.03 \Omega, r_{1}=r_{2}=0.47 \Omega$ while on the other was another converter with carefully selected asymmetric components based on the optimization equations derived in (62) by only modifying the optimization capacitor and inductor to $\mathrm{C}_{1}=80 \mu \mathrm{F}$ and $\mathrm{L}_{1}=4 \mu \mathrm{H}$ as shown in Table 1 . The new smaller values of $\mathrm{C}_{1}$ and $\mathrm{L}_{1}$ pushes the poles of $G_{\widetilde{V}_{\mathrm{g}}}^{\widetilde{V}_{\mathrm{C}}}, \mathrm{G}_{\widetilde{\mathrm{d}}}^{\widetilde{V}_{\mathrm{C} 1}}$ and $\mathrm{G}_{\tilde{\mathrm{I}}_{O}}^{\widetilde{\mathrm{V}}_{\mathrm{C} 1}}$ further away on the left hand plane (LHP).

Although the values of $R_{1}$ and $r_{1}$ are proportional to $C_{1}$ and $R_{1}$ respectively, and each can influence the position, the choice of smaller $\mathrm{L}_{1}$ and $\mathrm{C}_{1}$ are preferred due to the inefficiency associated with parasitic resistances and other constraints such as weight and size associated with larger capacitors and inductors. The new values of $\mathrm{C}_{1}$ and $\mathrm{L}_{1}$ also ensures that the poles of the TFs of $\tilde{\mathrm{v}}_{\mathrm{C} 2}$ and $\tilde{\mathrm{l}}_{\mathrm{L} 1}$ are real and non-positive instead of the complex pole that existed from $\mathrm{C}_{1}=400 \mu \mathrm{F}$ and $\mathrm{L}_{1}=500 \mu \mathrm{H}$. The response of the two circuits with respect to output voltage $\left(V_{O}\right)$, output current $\left(I_{O}\right)$ and input current $\left(I_{g}\right)$ are presented in Figure 3. Figure 4 (a) shows the ideal gain curve of the converter.

Their operations were also compared using ideal components by neglecting the parasitic resistances $R_{1}, R_{2}, r_{1}$ and $r_{2}$ for both the optimized and symmetric circuits in order to compare their output voltages with the ideal steady state output voltage of (7) and identify the effects of the parasitic resistances as shown in Figure 4(b) and (c).

$\underline{\text { Table 1. Parameter values used for simulation }}$

\begin{tabular}{ccc}
\hline \multirow{2}{*}{ Parameter } & \multicolumn{2}{c}{ Value } \\
& Symmetric & Optimized \\
\hline $\mathrm{V}_{\mathrm{g}}(\mathrm{V})$ & 12 & 12 \\
$\mathrm{D}$ & 0.63 & 0.63 \\
$\mathrm{f}(\mathrm{KHz})$ & 100 & 100 \\
$\mathrm{C}_{1}(\mu \mathrm{F})$ & 400 & 80 \\
$\mathrm{C}_{2}(\mu \mathrm{F})$ & 400 & 400 \\
$\mathrm{~L}_{1}(\mu \mathrm{H})$ & 500 & 4 \\
$\mathrm{~L}_{2}(\mu \mathrm{H})$ & 500 & 500 \\
$\mathrm{R}_{1}(\Omega)$ & 0.03 & 0.03 \\
$\mathrm{R}_{2}(\Omega)$ & 0.03 & 0.03 \\
$\mathrm{r}_{1}(\Omega)$ & 0.47 & 0.47 \\
$\mathrm{r}_{2}(\Omega)$ & 0.47 & 0.47 \\
$\operatorname{Load}(\Omega)$ & 7 & 7 \\
\hline
\end{tabular}

\section{RESULTS AND DISCUSSION}

Results of Figure 3 confirm the validity of these equations because the output voltage and output current of the optimized circuit are $15.25 \mathrm{~V}$ and $2.18 \mathrm{~A}$ against $13.15 \mathrm{~V}$ and $1.87 \mathrm{~A}$ obtained without optimization respectively. This is because the optimization capacitor $C_{1}$ and inductor $L_{1}$ were selected based on the equations derived from this model as discussed in the Analysis and Verification sections rather than symmetry. This increase represents a magnitude increase of $16.35 \%$ and $16.58 \%$ for the output voltage and output current 
respectively. The optimized outputs both have ripples of $<3 \%$. The wave shape of the input current changed from the previous pulsating square wave to saw-tooth after the optimization as shown in Figure 3(c).

Plot of the ideal gain against duty ratio of the converter obtained from (7) is shown in Figure 4(a). From (7), the magnitude of the ideal steady-state output voltage for this converter at input voltage $\left(\mathrm{V}_{\mathrm{g}}\right)$ of $12 \mathrm{~V}$ and duty ratio (D) of 0.63 is $20.43 \mathrm{~V}$. This is greater than the average output voltages of $15.25 \mathrm{~V}$ and $13.15 \mathrm{~V}$ obtained from simulation results of Figure 3(a) for both the optimized real and the symmetric real circuits respectively due to voltage drops across the parasitic resistances. The parasitic voltage drops are dependent on the magnitude of the parasitic resistances and the currents flowing in the circuits. This also shows that the optimized circuit has less parasitic voltage drop than the symmetric (non-optimized) circuit thus implying higher efficiency. This is further verified in Figure 4(b) and (c) where responses of the same circuits without parasitic resistances are also presented. The ideal circuits' steady-state responses in Figure 4(b) shows increased output voltages to about $20.43 \mathrm{~V}$ and $19.20 \mathrm{~V}$ due to the elimination of parasitic voltage drops. This implies that the response of the simulated ideal optimized circuit is the same as the ideal analytical output voltage magnitude of $20.43 \mathrm{~V}$ in (69), which is about $6.41 \%$ higher than the $19.20 \mathrm{~V}$ for the ideal symmetric circuit. This further verifies the validity of the model derived here.

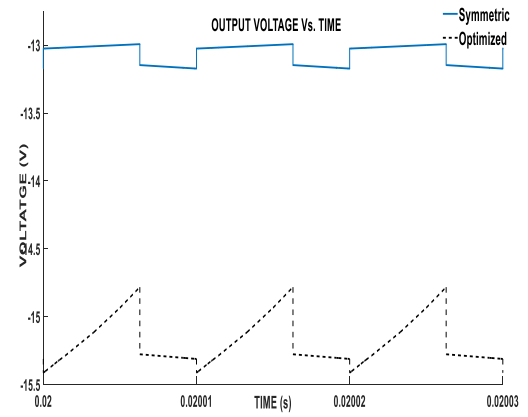

(a)

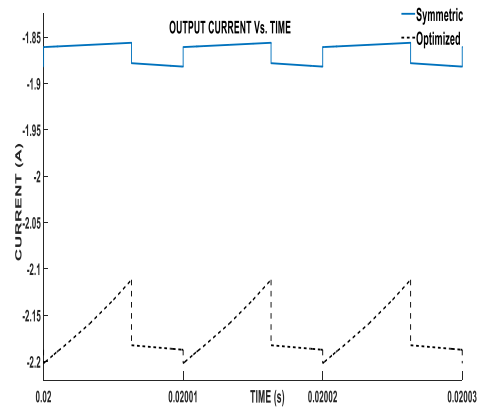

(b)

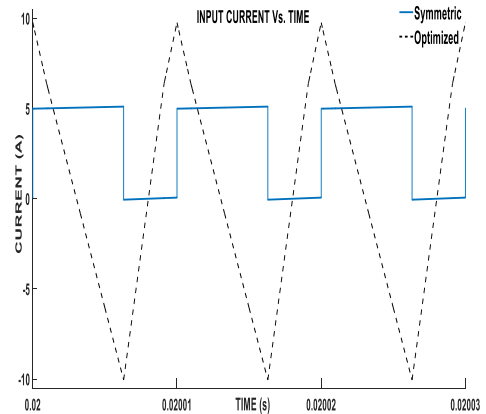

(c)

Figure 3. Simulation results of the same converter but different parameters with the solid blue line representing a symmetric circuit and the dashed black line representing a carefully selected (optimized) values based on this modelling (a) Output Voltage (b) Output Current (c) Input Current

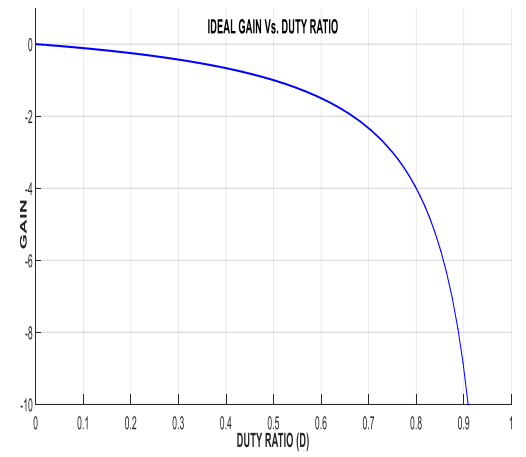

(a)

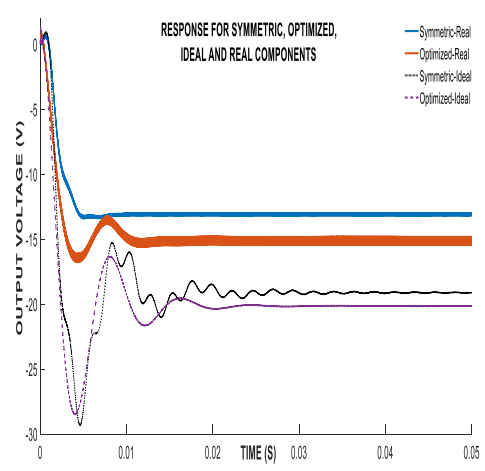

(b)

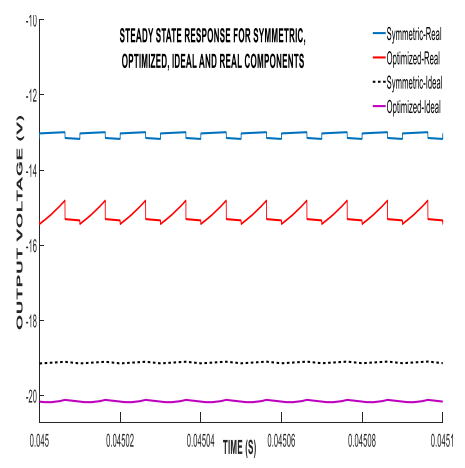

(c)

Figure 4. (a) Converter's ideal gain curve (b) Converter's transient response for $0>t<0.05 \mathrm{~s}$ using symmetricreal, optimized-real, symmetric-ideal and optimized-ideal components (c) Convert's steady state response using symmetric-real, optimized-real, symmetric-ideal and optimized-ideal components

\section{CONCLUSION}

A dynamic model of a DC-DC q-ZSC with buck-boost converter gain has been presented. The modelling considered non-symmetric non-ideal capacitors and inductors. The use of non-ideal components was fruitful because it was found that they have a significant effect on the poles and zero positions of most of the transfer functions. It was also found that similar to the existing impedance source converters, there may also exist right-hand plane (RHP) zero in the duty ratio to output capacitor voltage. It was also found and verified that rather than using symmetric components, use of carefully selected smaller asymmetric components produces 
less parasitic voltage drop, higher output voltage and current under the same conditions. This means better performance and efficiency at reduced cost, size and weight because smaller components could be used to achieve the required optimization ratios where applicable.

\section{REFERENCES}

[1] F. Z. Peng, “Z-Source Inverter,” 37th IEEE Ind. Appl. Conf., vol. 2, pp. 775-781, 2002.

[2] A. Chub, D. Vinnikov, F. Blaabjerg, and F. Z. Peng, "A review of galvanically isolated impedance-source DC-DC converters," IEEE Trans. Power Electron., vol. 31, no. 4, pp. 2808-2828, 2016.

[3] M. K. Nguyen, Y. C. Lim, and Y. J. Kim, “A modified single-phase quasi-Z-source ac-ac converter," IEEE Trans. Power Electron., vol. 27, no. 1, pp. 201-210, 2012.

[4] F. Z. Peng, X. Yuan, X. peng Fang, and Z. Qian, “Z-source inverter for adjustable speed drives," IEEE Power Electron. Lett., vol. 99, no. 2, pp. 33-35, 2003.

[5] X. P. Fang, Z. Qian, and F. Z. Peng, "Single-Phase Z-Source PWM AC-AC Converters," IEEE Power Electron. Lett., vol. 3, no. 4, pp. 121-124, 2005.

[6] X. Ding, Z. Qian, Y. Xie, and Z. Lu, "Three-Phase Z-Source Rectifier," in 36th IEEE Power Electronics Specialists Conference, 2005, pp. 494-500.

[7] D. Li, P. C. Loh, M. Zhu, F. Gao, and F. Blaabjerg, "Enhanced-boost Z-source inverters with alternate-cascaded switched-and tapped-inductor cells," IEEE Trans. Ind. Electron., vol. 60, no. 9, pp. 3567-3578, 2013.

[8] K. Beer and B. Piepenbreier, "Properties and advantages of the quasi-Z-source inverter for DC-AC conversion for electric vehicle applications," 2010 Emobility - Electr. Power Train, EEPT 2010, 2010.

[9] F. Guo, L. Fu, C. H. Lin, C. Li, W. Choi, and J. Wang, "Development of an 85-kW bidirectional quasi-Z-source inverter with DC-link feed-forward compensation for electric vehicle applications," IEEE Trans. Power Electron., vol. 28, no. 12, pp. 5477-5488, 2013.

[10] F. Z. Peng et al., "Z-Source Inverter for Motor Drives," IEEE Trans. Power Electron., vol. 20, no. 4, pp. 857-863, 2005.

[11] W. Mo, P. C. Loh, and F. Blaabjerg, “Asymmetrical Г-Source Inverters,” IEEE Trans. Ind. Electron., vol. 61, no. 2, pp. 637-647, 2014.

[12] D. Cao, S. Jiang, X. Yu, and F. Z. Peng, "Low-Cost Semi-Z-source Inverter for Single-Phase," IEEE Trans. Power Electron., vol. 26, no. 12, pp. 3514-3523, 2011.

[13] D. Cao and F. Z. Peng, "A Family of Z-source and Quasi-Z-source DC-DC Converters," 2009 Twenty-Fourth Annu. IEEE Appl. Power Electron. Conf. Expo., pp. 1097-1101, 2009.

[14] B. Zhao, Q. Yu, Z. Leng, and X. Chen, "Switched Z-source isolated bidirectional dc-dc converter and its phase-shifting shoot-through bivariate coordinated control strategy," IEEE Trans. Ind. Electron., vol. 59, no. 12, pp. 4657-4670, 2012.

[15] V. P. Galigekere and M. K. Kazimierczuk, "Analysis of PWM Z-source DC-DC converter in CCM for steady state," IEEE Trans. Circuits Syst. I Regul. Pap., vol. 59, no. 4, pp. 854-863, 2012.

[16] M. Egorov, D. Vinnikov, R. Strzelecki, and M. Adamowicz, "Impedance-Source Inverter-Based High-Power DC/DC Converter for Fuel Cell Applications," Proceeding 8th Int. Conf. Environ. Electr. Eng., no. 1, pp. 1-4, 2015.

[17] V. Vaisanen, T. Riipinen, J. Hiltunen, and P. Silventoinen, "Design of $10 \mathrm{~kW}$ resonant push-pull DC-DC converter for solid oxide fuel cell applications," in 14th European Conference on Power Electronics and Applications, 2011, pp. $1-10$.

[18] D. Vinnikov and I. Roasto, "Quasi-Z-Source-based isolated DC/DC converters for distributed power generation," IEEE Trans. Ind. Electron., vol. 58, no. 1, pp. 192-201, 2011.

[19] J. Zakis, D. Vinnikov, O. Husev, and I. Rankis, "Dynamic behaviour of qZS-based bi-directional DC/DC converter in supercapacitor charging mode," SPEEDAM 2012 - 21 st Int. Symp. Power Electron. Electr. Drives, Autom. Motion, pp. 764-768, 2012.

[20] H. Cha, F. Z. Peng, and D. W. Yoo, "Distributed impedance network (Z-network) DC-DC converter," IEEE Trans. Power Electron., vol. 25, no. 11, pp. 2722-2733, 2010.

[21] I. Roasto, D. Vinnikov, T. Jalakas, Z. Janis, and S. Ott, "Experimental Study of Shoot-Through Control Methods for qZSI-Based DC/DC Converters," Int. Symp. Power Electron. Electr. Drives, Autom. Motion, pp. 293-296, 2010.

[22] D. Vinnikov, I. Roasto, and T. Jalakas, "New Step-Up DC / DC Converter with High-Frequency Isolation," in 35th Annual Conference of IEEE Industrial Electronics, 2009, pp. 670-675.

[23] I. Roasto, D. Vinnikov, J. Zakis, and O. Husev, "New shoot-through control methods for qZSI-based DC/DC converters," IEEE Trans. Ind. Informatics, vol. 9, no. 2, pp. 640-647, 2013.

[24] Y. P. Siwakoti, F. Z. Peng, F. Blaabjerg, P. C. Loh, and G. E. Town, "Impedance-source networks for electric power conversion Part I: A topological review," IEEE Trans. Power Electron., vol. 30, no. 2, pp. 699-716, 2015.

[25] J. Anderson and F. Z. Peng, "Four quasi-Z-Source inverters," in 2008 IEEE Power Electronics Specialists Conference, 2008, pp. 2743-2749.

[26] S. Yang, X. Ding, F. Zhang, F. Z. Peng, and Z. Qian, “Unified Control Technique for Z-Source Inverter," in 2008 IEEE Power Electronics Specialists Conference, 2008, pp. 3236-3242.

[27] J. Anderson and F. Z. Peng, "A class of quasi-Z-source inverters," in Industry Applications Society Annual Meeting, 2008. 2008. IAS '08. IEEE, 2008, pp. 1-7, 2008, pp. 1-7.

[28] F. Z. Peng, M. Shen, and K. Holland, "Application of Z-Source Inverter for Traction Drive of Fuel Cell-Battery Hybrid Electric Vehicles,” IEEE Trans. Power Electron., vol. 22, no. 3, pp. 1054-1061, 2007. 
[29] M. Shen, Q. Tang, and F. Z. Peng, "Modeling and controller design of the Z-source inverter with inductive load," PESC Rec. - IEEE Annu. Power Electron. Spec. Conf., pp. 1804-1809, 2007.

[30] X. Ding, Z. Qian, S. Yang, B. Cui, and F. Z. Peng, "A high-performance Z-source inverter operating with small inductor at wide-range load," Conf. Proc. - IEEE Appl. Power Electron. Conf. Expo. - APEC, pp. 615-620, 2007.

[31] M. Shen, J. Wang, A. Joseph, F. Z. Peng, L. M. Tolbert, and D. J. Adams, "Maximum constant boost control of the Zsource inverter," 39th IEEE/IAS Ind. Appl. Conf., vol. 1, no. I, pp. 142-147, 2004.

[32] U. Supatti and F. Z. Peng, "Z-source inverter based wind power generation system," 2008 IEEE Int. Conf. Sustain. Energy Technol., pp. 634-638, 2008.

[33] Y. Li, J. Anderson, F. Z. Peng, and D. Liu, "Quasi-Z-Source Inverter for Photovoltaic Power Generation Systems," Appl. Power Electron. Conf. Expo. 2009. APEC 2009. Twenty-Fourth Annu. IEEE, pp. 918-924, 2009.

[34] M. Shen and F. Z. Peng, "Operation modes and characteristics of the Z-source inverter with small inductance or low power factor," IEEE Trans. Ind. Electron., vol. 55, no. 1, pp. 89-96, 2008.

[35] M. Shen, A. Joseph, Y. Huang, F. Z. Peng, and Z. Qian, "Design and Development of a 50kW Z-Source Inverter for Fuel Cell Vehicles," in 5th ICES/EEE International Power Electronics and Motion Control Conference, 2006.

[36] S. Yang, Q. Lei, F. Z. Peng, R. Inoshita, and Z. Qian, "Current-fed quasi-Z-source inverter with coupled inductors," 2009 IEEE Energy Convers. Congr. Expo. ECCE 2009, pp. 3683-3689, 2009.

[37] Y. Huang, M. Shen, F. Z. Peng, and J. Wang, "Z-source inverter for residential photovoltaic systems," IEEE Trans. Power Electron., vol. 21, no. 6, pp. 1776-1782, 2006.

[38] F. Z. Peng, "Z-source inverter," IEEE Trans. Ind. Appl., vol. 39, no. 2, pp. 504-510, 2003.

[39] P. C. Loh, D. M. Vilathgamuwa, Y. Sen Lai, G. T. Chua, and Y. Li, "Pulse-width modulation of Z-source inverters," IEEE Trans. Power Electron., vol. 20, no. 6, pp. 1346-1355, 2005.

[40] J. Liu, J. Hu, and Longya Xu, "A Modified Space Vector PWM for Z-Source Inverter - Modeling and Design," in 8th International Conference on Electrical Machines and Systems, 2005., 2005, vol. 2, pp. 1242-1247.

[41] J. Liu, J. Hu, and L. Xu, "Dynamic Modeling and Analysis of Z Source Converter - Derivation of AC Small Signal Model and Design-Oriented Analysis," IEEE Trans. Power Electron., vol. 22, no. 5, pp. 1786-1796, 2007.

[42] C. J. Gajanayake, D. M. Vilathgamuwa, and P. C. Loh, "Development of a comprehensive model and a multiloop controller for Z-source inverter DG systems,” IEEE Trans. Ind. Electron., vol. 54, no. 4, pp. 2352-2359, 2007.

[43] Y. Liu, B. Ge, F. J. T. E. Ferreira, A. T. De Almeida, and H. Abu-Rub, "Modeling and SVPWM control of quasi-Zsource inverter," Proceeding Int. Conf. Electr. Power Qual. Util. EPQU, no. 09, pp. 95-101, 2011.

[44] Y. Li, S. Jiang, J. G. Cintron-Rivera, and F. Z. Peng, "Modeling and control of quasi-z-source inverter for distributed generation applications," IEEE Trans. Ind. Electron., vol. 60, no. 4, pp. 1532-1541, 2013.

[45] C. J. Gajanayake, D. M. Vilathgamuwa, and P. C. Loh, "Small-signal and signal-flow-graph modeling of switched Zsource impedance network," IEEE Power Electron. Lett., vol. 3, no. 3, pp. 111-116, 2005.

[46] R. D. Middlebrook and S. Cuk, "A General Unified Approach to Modelling Switching-Converter Power Stages," in IEEE Power Electronics Specialists Conference, 1976, pp. 18-34.

[47] D. W. Hart, Power Electronics. New York: McGraw-Hill, 2011.

[48] P. C. Loh, D. M. Vilathgamuwa, C. J. Gajanayake, Y. R. Lim, and C. W. Teo, "Transient modeling and analysis of pulse-width modulated Z-source inverter," IEEE Trans. Power Electron., vol. 22, no. 2, pp. 498-507, 2007.

[49] S. Skogestad and I. Postletwaite, Multivariable Feedback Control Analysis and design. New York: John Wiley \& Sons, 1996.

[50] A. M. Florez-Tapia, F. M. Ibanez, J. Vadillo, I. Elosegui, and J. M. Echeverria, "Small signal modeling and transient analysis of a Trans quasi-Z-source inverter," Electr. Power Syst. Res., vol. 144, pp. 52-62, 2017.

[51] J. Khajesalehi, M. Hamzeh, K. Sheshyekani, and E. Afjei, "Modeling and control of quasi Z-source inverters for parallel operation of battery energy storage systems: Application to microgrids," Electr. Power Syst. Res., vol. 125, pp. 164$173,2015$.

[52] V. P. Galigekere N. and M. K. Kazimierczuk, "Small-signal modeling of open-loop PWM Z-source converter by circuit-averaging technique," IEEE Trans. Power Electron., vol. 28, no. 3, pp. 1286-1296, 2013.

[53] M. Ado, A. Jusoh, A. U. Mutawakkil, and S. M. Ayob, "Two q-ZSCs with Efficient Buck-Boost Gain," in 10th IEEE PES Asia-Pacific Power and Energy Engineering Conference, 2018, pp. 252-256.

[54] M. Ado, A. Jusoh, M. J. A. Aziz, M. Kermadi, and A. U. Mutawakkil, "DC-DC q-ZSC with Buck-Boost Converter Gain," in 9th IEEE Control and System Graduate Research Colloquium, 2018, pp. 86-89.

[55] M. Ado, A. Jusoh, S. M. Ayob, M. H. Ali, and G. S. M. Galadanchi, "Buck-Boost Converter with q-ZSC Topology," in 5th IET International Conference on Clean Energy and Techonology, 2018.

[56] R. W. Erickson, Fundamentals of Power Electronics with MATLAB. 2007. 